\title{
Real-Time Imaging of Accessible Axon Guidance Assays in Three-Dimensional Culture
}

\author{
Daniel Terheyden-Keighley ${ }^{1}$, Beate Brand-Saberi ${ }^{2}$ and Carsten Theiss ${ }^{{ }^{*}}$ \\ ${ }^{1}$ Institute of Anatomy, Department of Cytology, Ruhr-University Bochum, Universitätsstraße 150, 44780 Bochum, Germany \\ ${ }^{2}$ Institute of Anatomy, Department of Molecular Embryology, Ruhr-University Bochum, Universitätsstraße 150, 44780 Bochum, Germany
}

\author{
Correspondence to: \\ Dr. Carsten Theiss \\ Institute of Anatomy \\ Department of Cytology, Ruhr-University \\ Bochum, Universitätsstraße 150 \\ D-44780 Bochum, Germany \\ Tel: (+49) (0)234 32-24560 \\ E-mail: carsten.theiss@rub.de
}

Received: November 26, 2016

Accepted: January 04, 2017

Published: January 05, 2017

Citation: Terheyden-Keighley D, Brand-Saberi B, Theiss C. 2017. Real-Time Imaging of Accessible Axon Guidance Assays in Three-Dimensional Culture. JNeurol Exp Neurosci 2(2): 34-39.

Copyright: (C) 2017 Terheyden-Keighley et al. This is an Open Access article distributed under the terms of the Creative Commons Attribution 4.0 International License (CC-BY) (http:// creativecommons.org/licenses/by/4.0/) which permits commercial use, including reproduction, adaptation, and distribution of the article provided the original author and source are credited.

Published by United Scientific Group

\begin{abstract}
During the development of the peripheral nervous system, neurons rely on precise timing and extra-cellular signalling to guide their axons to exact locations. This process is most readily apparent when looking at sensory and motor neurons from in and around the spinal cord, and how they project their axons deep into the periphery. These developmental guidance systems are inactive in the adult, leaving the peripheral nervous system to rely on imprecise regeneration mechanisms to recover from injury. To study these embryonic guidance processes, in vitro guidance models of ever increasing complexity and cost have been devised. Here, we demonstrate three accessible, yet effective protocols for testing the effects of signalling factor gradients on embryonic chicken sensory neurons in three-dimensional culture. These range from bead-based point sources, to diffusion-based microfluidics chambers and fluorescence-based live cell imaging via spinning disc confocal microscopy. Basic research on axon guidance will be critical for exploiting their mechanisms in neuroregenerative medicine.
\end{abstract}

\section{Keywords}

In vitro, Model, 3D culture, Collagen gel, Electroporation, Sensory neurons, DRG, Microfluidics, Bead, Chicken

\section{Introduction}

The peripheral nervous system has a higher capacity for regeneration after injury than the central nervous system. Even so, peripheral regeneration is still liable to fail. Unaided recovery after a major physical trauma, such as $4^{\text {th }}$ or $5^{\text {th }}$ degree injuries in the Sunderland classification system, is currently unrealistic [1]. The current gold standard for heavy nerve trauma is nerve grafting, which is the process of transplanting a nerve of lesser importance into the site of damage to act as a guide for the regenerating axons [2]. This relatively simple, yet inelegant method results in the sure destruction of one area, for only the chance of partial recovery in another. Through the study of axon guidance mechanisms, these embryonic processes may one day be implemented in the targeted regeneration of damaged nervous tissue.

In the event of peripheral nerve damage, degeneration occurs in both anterograde and retrograde directions from the point of lesion, known as Wallerian degeneration [3]. While anterograde degeneration results in Schwann cells and macrophages clearing the distal part of the axon, retrograde degeneration only proceeds up to the next node of Ranvier. The Schwann cells lining the distal axon stub transdifferentiate from myelinating Schwann cells into regenerative Schwann cells, phagocytizing myelin and axon remnants, followed by the 
maintenance of a guiding 'tunnel' [4]. At this point, axon sprouting is initiated, with multiple processes erupting from the proximal stub. The intracellular detection and regulation of this process is initiated by importin proteins complexing with dynein and transporting transcription factors into the nucleus, activating the various genes required for regrowth [5]. These growth cones then grow along inside the basal laminar tubes (also known as bands of Büngner, and are maintained by the Schwann cells) at a speed of up to $3 \mathrm{~mm}$ per day in an attempt to re-innervate their target structures [6]. This is where complications can occur, specifically when the remnants from the previous nerve are missing, such as after heavy trauma, preventing the newly sprouted growth cones from finding their target. Regeneration is also impaired in situations where the distal stub is too far away from the proximal stub, such that the regenerating axon cannot find a basal laminar tube to grow into. In the mouse model, a critical window of around 35 days exists, within which a regenerating motor neuron must reach its target muscle, lest it become unreceptive [7]. Even in situations where a regenerating axon manages to innervate a target, the non-specificity of the regeneration progress can result in the innervation of a totally different muscle (in the case of motor neurons) [8].

The regeneration of organs and tissues can be thought of in terms of a specific adult stem cell population becoming active, then generating the necessary cell types that then go on to multiply and migrate to reconstitute the tissue. Well-known examples of which include: blood, fat, muscle and various epithelia [9-11]. This stem cell-centric approach has many parallels with embryonic development, however regeneration in the mammalian peripheral nervous system takes a different approach. The peripheral nervous system's response to damage is repair on a sub-cellular level, i.e. regrowing the distal axon process. This implies that different processes will re-establish the connections.

A number of factors have been categorized as being vital during neural development. These classical neurotrophic factors play various roles in axon chemoattraction, growth rate, differentiation and survival [12]. Two well-known examples of these are Nerve Growth Factor (NGF) and Brain-derived Neurotrophic Factor (BNDF), which are diffusible factors that act over a large distance. Neurotrophic factors work in a concentration-dependent manner, with their chemotactic effects being dependent upon reacting to the directionality of the concentration gradient. BDNF, for example, has been shown to be a long-range attractive factor for growth cones of certain sensory neurons in the dorsal root ganglia, of which only around $30 \%$ are sensitive to BNDF via the expression of the Tropomyosin receptor kinase $\mathrm{B}(\operatorname{TrkB})$ receptor [13].

The canonical neurotrophic factor is NGF, or Nerve Growth Factor. The first indication of its chemoattractive properties came about using a Campenot chamber [14]. This chamber separated neurons from a solution of NGF using dividers with thin gaps under them, setting up an attractive gradient. Tissue co-culture models have also been used to demonstrate chemoattractive properties, unfortunately there is no signalling factor concentration control [15].
Microfluidic flow chambers facilitate the controlled, active flow of liquid over neurons and their neurites. These chambers are limited to 2D culture, and thus suffer from shear stresses caused by the force of the liquid moving over the neurons. It has been shown that even moderate flow rates have a detrimental effect on neuronal culture [16]. New methods employ ever more complex chambers in an attempt to reduce shear stress, however this also reduces their accessibility and increases their cost. In a similar way, micro-pipettes have been used as a point source where a test molecule is slowly injected into the medium. These are set up so as to point the stream at a $45^{\circ}$ angle from the axon's longitudinal axis, and then observe the axon's growth direction. The primary disadvantage here is the single neuron restriction [17]. Other issues include the steady accumulation of the injected factor, limited growth distance before needing to readjust the pipette, and maintaining sterile culture conditions of an open dish.

Collagen gel mimics the extra-cellular matrix (ECM) in which cells are embedded, bringing in vitro models closer to the in vivo conditions they are intended to replicate. Three-dimensional culture has been shown to be crucial for the intrinsic properties of various cell types, including multipotency, cell type stability, and basic morphology [18]. When looking specifically at neurons, the classical axon bifurcation phenotype of sensory neurons in the dorsal root ganglia (DRG) is absent when cultured on a $2 \mathrm{D}$ substrate, whereas in 3D culture it can be clearly seen [19]. Replicating the actual environment in which growth cones navigate is absolutely crucial for modelling their response to biological factors. Emulating the ECM is especially important when creating assays for long distance signalling, as it is through the ECM that many signalling molecules diffuse. In addition to stabilizing signalling molecule gradients, collagen gels allow for the precise placement of both test cells and signalling sources, such as heparin-acrylic beads soaked in a moleculeof-interest. The test cells can be in the form of an organotypic culture such as an entire DRG/spinal cord explant, or they can be in the form of dissociated cells mixed into the gel before it polymerizes. The bead-based approach has the advantage of being directly comparable to in vivo experiments where the same beads are inserted into a developing chicken embryo to see its effect after continued development [20].

In this work, we will detail and demonstrate new methods which allow for the quantification of the axon guidance properties of a molecule-of-interest on various neuronal sources. This includes the quantification of the concentration sensitivity range, chemoattractive/repulsive properties of axon/ cell migration, and finally axon growth rate. To achieve these aims, state-of-the-art technologies and culture methods such as live-cell spinning disc confocal microscopy, microfluidic chambers and 3D culture are combined to produce effective axon guidance assays.

\section{Materials and Methods}

All animal experiments in this study have been performed in strict accordance with institutional, German (TschG), and EU guidelines for the care and use of laboratory animals. 


\section{Explantation of chicken DRGs}

Fertilized chicken eggs from a local breeder were incubated for between 5 and 11 days, depending on the desired embryonic stage. The incubation chamber was held at $37{ }^{\circ} \mathrm{C}$ and $70 \%$ humidity, with the eggs being turned once per day. On the day of explantation, 10-20 eggs of the desired stage were carefully cracked open, and the embryos removed using a perforated spoon and forceps (Figure 1A and 1B). Embryos were collected in a petri dish containing ice-cold Hank's balanced salt solution (HBSS), after which they were rinsed in additional dishes of HBSS to remove traces of yolk and any remaining fragments of extra-embryonic tissue (Figure $1 \mathrm{C})$. Following this, the embryos were roughly dissected using curved forceps to remove the tail, peritoneal organs and fat deposits to expose the developing spine and peripheral DRGs in the sacro-lumbar region (Figure 1D). Under a dissecting microscope, DRGs were carefully excised using a pair of ultrafine forceps. This was done by gently pulling the thigh away from the spine, clearly exposing the dorsal entry tracts of the sacro-lumbar DRGs, allowing them to be severed (Figure 1E and $1 \mathrm{~F})$. Next, individual DRGs were plucked away from the plexus lumbar-sacralis, and transferred into a small volume of HBSS. Using this method, 8-10 DRGs were extracted per embryo, for a total of up to 200 DRGs gathered in a single procedure (Figure $1 \mathrm{G}$ ).

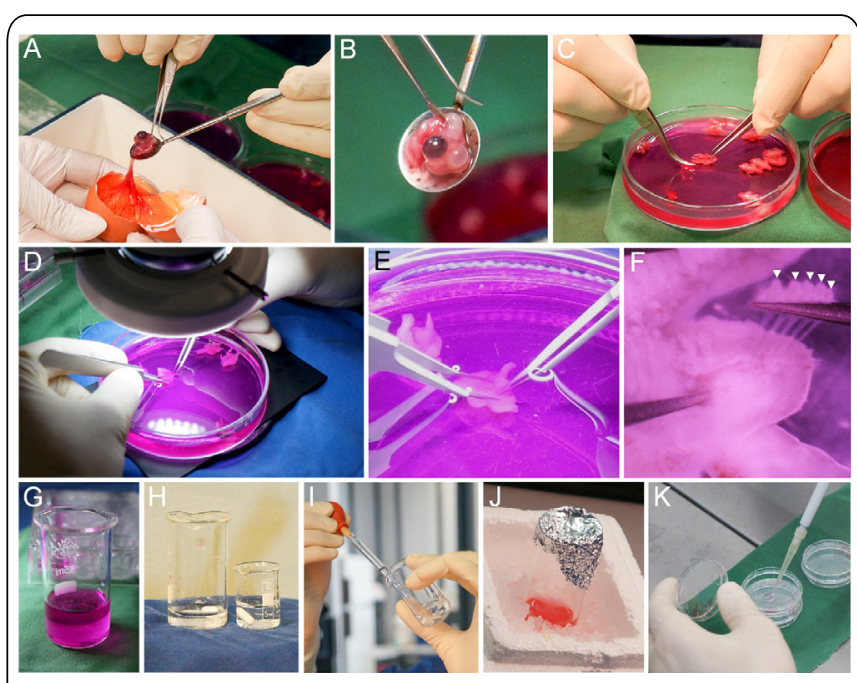

Figure 1: Chicken DRG harvest procedure (A) Assistant cracks open egg with a standard tea spoon and presents the embryo. (B) Curved forceps hook the neck, allowing the embryo to be lifted out on a perforated spoon. (C) The head is discarded, followed by removal of the internal organs. (D) Under a dissecting microscope, fatty connective tissue is removed from around the ganglia. (E) Tension is applied between the thigh and spine to reveal the dorsal/ventral root nerves, which are then severed with ultra-fine forceps. (F) Ganglia are plucked from their respective nerves and $(\mathbf{G})$ deposited in ice-cold HBSS. (H) Trypsination of ganglia with the aid of a magnetic stirrer and (I) pasture pipette titration. (J) Trypsination reaction stopped by transferring the cells into a centrifuge tube containing ice-cold serum-containing MEM medium. (K) After centrifugation, the cells are resuspended in ganglion medium, and then mixed into a freshly prepared collagen gel.

\section{Trypsination}

The collected DRGs were titrated in a $0.05 \%$ trypsin (Thermo Fischer Scientific, Darmstadt, Germany) in phosphate buffered saline (PBS) solution. This involved suspending the DRGs in $2.5 \mathrm{ml}$ trypsin solution for 5 minutes (agitated via magnetic stirrer), after which the DRGs were allowed to sediment, and the single cells/trypsin supernatant carefully transferred into $20 \mathrm{ml}$ of ice cold minimal essential medium (MEM, M2279, Sigma-Aldrich) supplemented with 10\% horse serum (S9135; Biochrom), 2 mM L-glutamine (G7513, Sigma-Aldrich), and penicillin (1000 unit/ml) + streptomycin (0.1 ng/ml) (P0781, Sigma-Aldrich) (Figure 1H-1J). This process was repeated 4 times, accompanied by gentle titration with a fire-polished Pasteur pipette at the start of the final round to help mechanically break apart any remaining chunks. The final cell suspension was then centrifuged at $2400 \mathrm{~g}$ for 10 minutes, after which the pellet was finally resuspended in a volume and liquid appropriate for the next procedure.

\section{NEON electroporation}

Trypsinated cells were electroporated with plasmids encoding fluorescent proteins using the $10 \mu 1$ NEON system (MPK1025, Invitrogen) to enable Z-stack time-lapse image capture. Following trypsination, the cells were washed by resuspension in $\mathrm{Mg}^{++} / \mathrm{Ca}^{++}$free PBS. Cell count and viability were assessed after washing with the aid of trypan blue (T8154, Sigma-Aldrich). The cell suspension was centrifuged again and resuspended in an amount of "Buffer R" appropriate for the number of electroporations to be carried out and the desired cell density. The plasmid is introduced to this mixture with a final concentration of $1 \mu \mathrm{g}$ per $10 \mu \mathrm{l}$ electroporation, but with the total plasmid solution added being less than $10 \%$ of the final volume. Using the specialized electrode pipette tips and pulse generator, the cells were exposed to an electric pulse with the following settings: $1300 \mathrm{v}, 20 \mathrm{~ms}$ pulse duration, 1 pulse. These cells were then directly added to $40 \mu 1$ of recovery media (MEM supplemented with 10\% horse serum, 0.6\% glucose, $1 \%$ chicken embryo extract, $2 \mathrm{mM}$ L-glutamine, 50 $\mathrm{ng} / \mathrm{ml}$ nerve growth factor-7S (N0513, Sigma-Aldrich)) for 5 minutes before being added to freshly made gel.

\section{Collagen gel culture preparation}

Collagen gels for 3D culture were prepared on ice using type I collagen (C7661, Sigma-Aldrich) in $0.1 \%$ acetic acid (A6283, Sigma-Aldrich). A standard gel would be made by first adding $5 \mu \mathrm{l}$ of $1 \mathrm{M} \mathrm{NaOH}$ to $28 \mu \mathrm{l}$ of $10 \mathrm{x}$ PBS (D1408, Sigma-Aldrich). To this, $250 \mu \mathrm{l}$ of $2 \mathrm{mg} / \mathrm{ml}$ collagen was added while carefully avoiding the introduction of air bubbles. $50 \mu \mathrm{l}$ of cell suspension (at the appropriate cell density) was then mixed in, after which $50 \mu \mathrm{l}$ droplets of the cell/gel mixture was then placed onto the centre of a $35 \mathrm{~mm}$ petri dish (Figure $1 \mathrm{~K})$. These dishes were then incubated at $37^{\circ} \mathrm{C} / 5 \% \mathrm{CO}_{2}$ for 30-40 minutes to facilitate polymerization, followed by the addition of $2 \mathrm{ml}$ of ganglion medium (MEM supplemented with $10 \%$ horse serum, $0.6 \%$ glucose, $1 \%$ chicken embryo extract, $2 \mathrm{mM}$ L-glutamine, $50 \mathrm{ng} / \mathrm{ml}$ nerve growth factor$7 \mathrm{~S}$ and penicillin $(1000 \mathrm{unit} / \mathrm{ml})+$ streptomycin $(0.1 \mathrm{ng} / \mathrm{ml}))$ to each dish. Heparin-acrylic beads (H5263, Sigma-Aldrich) soaked in various concentrations of test factor were implanted by first rinsing the beads in $1 \mathrm{ml} \mathrm{PBS}$, then injecting one or more of them into the centre of the gel in a $1 \mu \mathrm{l}$ PBS volume using a micropipette. 


\section{Chemotaxis chamber culture}

The chemotaxis chamber ( $\mu$-slide chemotaxis chamber, 80326 , Ibidi) is used for monitoring the whole-cell migration of cells, however here it was repurposed for monitoring axon outgrowth in the presence of factors (Figure 2A). In brief: All chamber ports are sealed other than those of the viewing chamber. $6 \mu$ of non-polymerized cell/gel mixture is introduced to one port, while the same volume is aspirated from the opposite side, drawing the liquid into the viewing chamber. These ports are then sealed, and the gel allowed to polymerize at $37^{\circ} \mathrm{C}$ for 30 minutes. Following this, the left and right media reservoirs are filled with the appropriate medium by unsealing the respective reservoir and injecting $65 \mu \mathrm{l}$ of medium. To set up a factor gradient, a $15 \mu \mathrm{l}$ droplet of factor-containing medium is applied to one port, while 15 $\mu \mathrm{l}$ of neutral medium is aspirated from the opposite port, this is done twice.

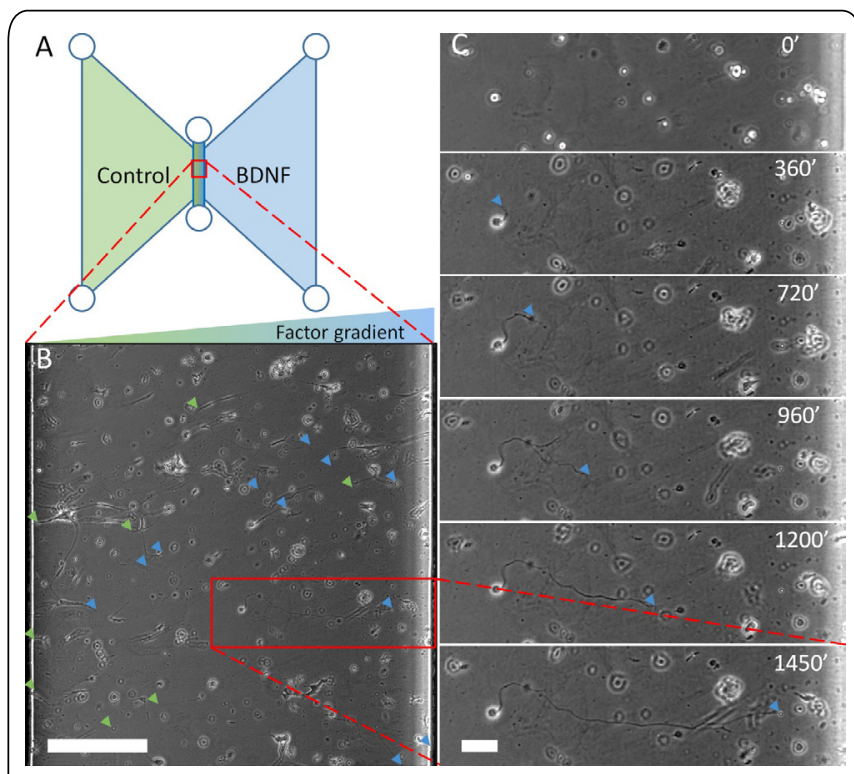

Figure 2: Chemotaxis gradient-chamber live cell imaging (A) Schematic representation of the Ibidi $\mu$-slide chemotaxis chamber - blue and green indicate test factor-containing media and control media respectively. The viewing chamber cross section is $1 \mathrm{~mm}$ wide, but only $70 \mu \mathrm{m}$ in height, keeping axons in a single focus plane. The central viewing chamber is $2 \mathrm{~mm}$ in length. (B) Topographical view of a portion of the viewing chamber, with test factor gradient indicated above. Growth cones travelling left are depicted with green arrow heads, whereas growth cones travelling to the right are marked with blue arrow heads. The expected $30 \%$ of sensory neuron BDNF sensitivity bias is observed. (C) A time-lapse series depicting the growth cone behaviour of a sensory neuron exposed to a gradient of BDNF (from the right side). Blue arrows indicate lead growth cone.

Scale bar:(B) $250 \mu \mathrm{m}$; (C) $50 \mu \mathrm{m}$

\section{Fixation and staining}

Immunostaining was used to label axons with antibodies against the light chain of neurofilament (NF-L). First, the collagen gel cultures were fixed in 4\% PFA in PBS for 20 minutes, followed by a washing step. The washing step consists of $3 \mathrm{x}$ short rinses, followed by $3 \mathrm{x}$ ten minute soaks in PBS. The cells were then permeablised by incubation with $0.015 \%$ Triton-X (T8532, Sigma-Aldrich) in PBS for five minutes at room temperature. After another wash step, the cells were blocked by incubation with $15 \%$ goat serum in PBS for 30 minutes, which was then directly replaced by the anti-NF-L antibody solution (mouse, 1:1000 in PBS, ab7255, abcam). This was then incubated in a dark, damp box over night at $4{ }^{\circ} \mathrm{C}$. After another wash step, the secondary antibody solution was applied (anti-mouse IgG TRITC 1:2000, T5393, Sigma-Aldrich) and incubated in a damp, dark box at room temperature for five hours. The gels were then subject to a final wash step before being submerged in PBS for imaging.

\section{Imaging}

Z-stack staged imaging of immunostained cultures, and live cell imaging was performed using a Nikon spinning disc confocal microscope with its PlanApo 10x (NA 0.25) and 20x (NA 0.75) objectives (CSU-W1, Visitron Systems, Germany). Additionally, the setup included a humidified, temperature regulated chamber held at $37^{\circ} \mathrm{C} / 5 \% \mathrm{CO}_{2}$ (Figure 3A-3D).

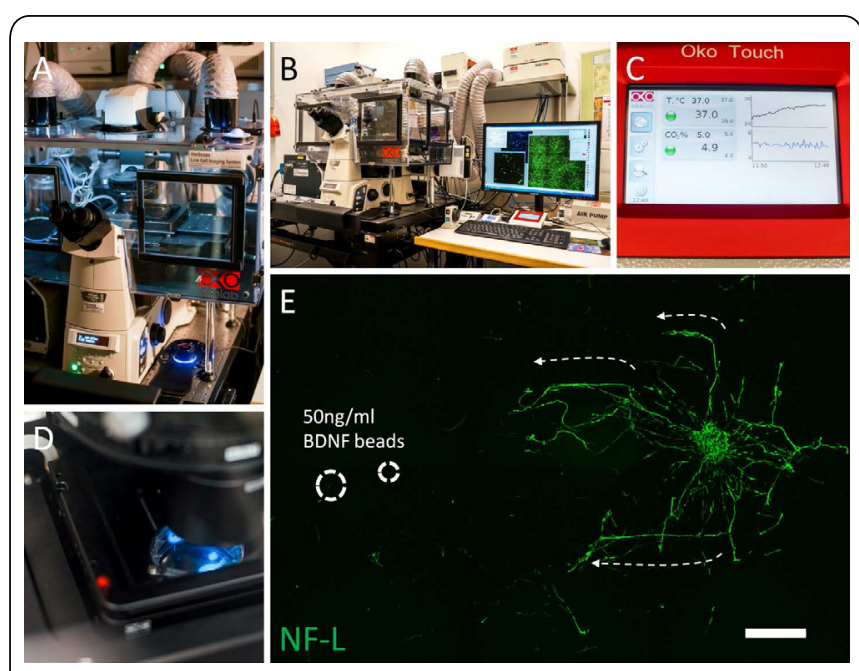

Figure 3: Spinning disc confocal microscope imaging (A) Complete with motorized stage and piezo-controlled fine-focus. (B) Pneumatic table for vibration isolation. (C) Incubation chamber for live-cell imaging including temperature and $\mathrm{CO}_{2}$ control. (D) Humidified sample chamber for reduced evaporation. (E) Positive control for axon guidance using BDNF-soaked beads (dashed circles, soaked overnight in $50 \mathrm{ng} / \mathrm{ml} \mathrm{BDNF}$ solution) as a known attractive factor for sensory neurons. Fragmented ganglia chunks fixed and immunostained against the neurofilament light chain after three days of culture.

Scale bar: $350 \mu \mathrm{m}$.

\section{Results and Discussion}

The post-mitotic nature of neurons has thus far prevented the easy culturing of these cells. To get around this issue, cell culture-based neuroscience has relied on the generation of primary neuronal cultures by directly harvesting the neurons from the embryos of various model animals. To this end, the chicken embryo offers one of the more versatile systems for acting as both a highly accessible neuronal source, and one which can be manipulated in ovo, with continued embryonic development afterwards. Chicken embryos can also be predictably and consistently produced due to our ability to pause their development by refrigeration after fertilization. In the peripheral nervous system, extracting neurons from their natural embryonic environment poses the challenge of distinguishing between real developmental processes, or the Wallerian degeneration repair mechanisms that are evident in the adult. Logically, neurons from an embryonic stage that is 
closer to the developmental time point of the nervous system will express more relevant developmental proteins than a more mature stage. To combat these challenges, this protocol balances the ideals of having the youngest possible embryos, with the least time required to harvest a desired cell population. For chicken dorsal root ganglia, this ideal range spans from embryonic day five to day ten (E5-10). While earlier stages are possible, extraction becomes drastically more difficult due to the ganglia being embedded in the surrounding mesoderm, as opposed to being free-standing structures. To compound the issue, the lack of contrast or pigmentation makes isolating only ganglionic cells very difficult. Here, we detail three accessible in vitro axon guidance models designed to apply gradients of a test molecule to the various forms of the harvested chicken ganglia to assess the response of the sensory neurons.

\section{Sensory neurons cultured with factor-soaked beads}

Explanted ganglia that were disassociated into single cells, broken into chunks or seeded as whole ganglia were seeded into collagen gel along with a test molecule-soaked bead. Following three days of incubation, immunostaining with neurofilament antibodies unveiled a clear chemoattractive response in a subset of DRG neurons exposed to a bead soaked in $50 \mathrm{ng} / \mathrm{ml} \mathrm{BDNF}$, reflecting the approximately $30 \%$ of DRG neurons expected to be BDNF sensitive [13] (Figure 3E). This also highlights the model's ability to easily test multiple concentrations in parallel: by first using soak-concentrations an order of magnitude apart (5, 50, 500 and $5000 \mathrm{ng} / \mathrm{ml})$, and then using a narrower range in the second round. The major advantage of the bead approach is its simplicity, with the bead behaving as a sponge, and thus acting as a point source to establish a radial gradient. The data were collected by a confocal spinning disc microscope, as a $50 \mu \mathrm{l}$ gel has an area of approximately $1 \mathrm{~cm}^{2}$ and thus requires that many pictures be taken rapidly. The $3 \mathrm{D}$ nature of axon growth through the gel dictates that a $Z$-stack of images needs to be combined to achieve a complete topographical image. This, combined with a staging function to scan the whole area could lead to photobleaching effects if done with a slower, conventional, laser scanning confocal microscope. A disadvantage is that the steepness of the gradient diminishes over time, remaining effective within three days (Figure 3E). Additionally, bead experiments can be directly replicated in vivo via chicken embryo bead implantation experiments using the exact same beads [20]. This would have the advantage of already having a perfectly working negative control model (a developing embryo), such that any effects on the nervous system can be observed in the context of its natural surroundings.

\section{Disassociated DRG microfluidics chamber culture}

By using collagen gels, the signalling molecules can only diffuse. This property of collagen gel had made it difficult to combine their use with flow-based microfluidics chambers [17]. However, by using a diffusion-based microfluidics chamber, test cells can be presented a binary choice in terms of axon growth direction. In these experiments, a chemotaxis chamber was repurposed as an axon guidance model system for use with disassociated DRG neurons. Briefly, it is composed by two chambers divided by a thin strip of collagen gel seeded with disassociated DRG cells. The triangle shape of the media chambers, combined with the very thin, collagen gel-filled viewing chamber, act together to restrict molecular movement, resulting in a stable and steep gradient over the neurons. To demonstrate this, one chamber was loaded with BNDF-containing medium, with the opposite chamber containing control medium. Here we see the expected $~ 30 \%$ of the sensory neurons being biased towards the BDNF gradient (in the chamber on the right side), as can be seen by the majority of growth cones pointing in that direction (Figure 2B). Due to the minimal thickness of the cell viewing chamber $(70 \mu \mathrm{m})$, the axons are constrained along the $\mathrm{Z}$-axis, allowing for growth cone tracking using a phase-contrast microscope (Figure 2B and 2C). Gradients established across the viewing chamber should be evenly distributed and stable for over 48 hours [21]. Optionally, this method can also be combined with genetic transformation to examine growth cone dynamics in the context of a stable signalling molecule gradient.

\section{Disassociated DRG transformation and live cell imaging}

The final approach combines imaging using a spinning disc confocal microscope, with disassociated DRG neurons electroporated with an enhanced-green fluorescent protein plasmid using an electroporation system to enable 3D livecell imaging. An optimization procedure revealed that the maximal transformation efficiency was at $1500 \mathrm{v}$ with a single $20 \mathrm{~ms}$ pulse, while the optimal neuron survival was achieved at 1300 v. Keeping the cell density within a narrow range proved crucial. Transfected cells were seeded into gels and allowed to polymerize. After 6 hours of incubation, fluorescent cells could be identified, allowing for the implantation of signalling

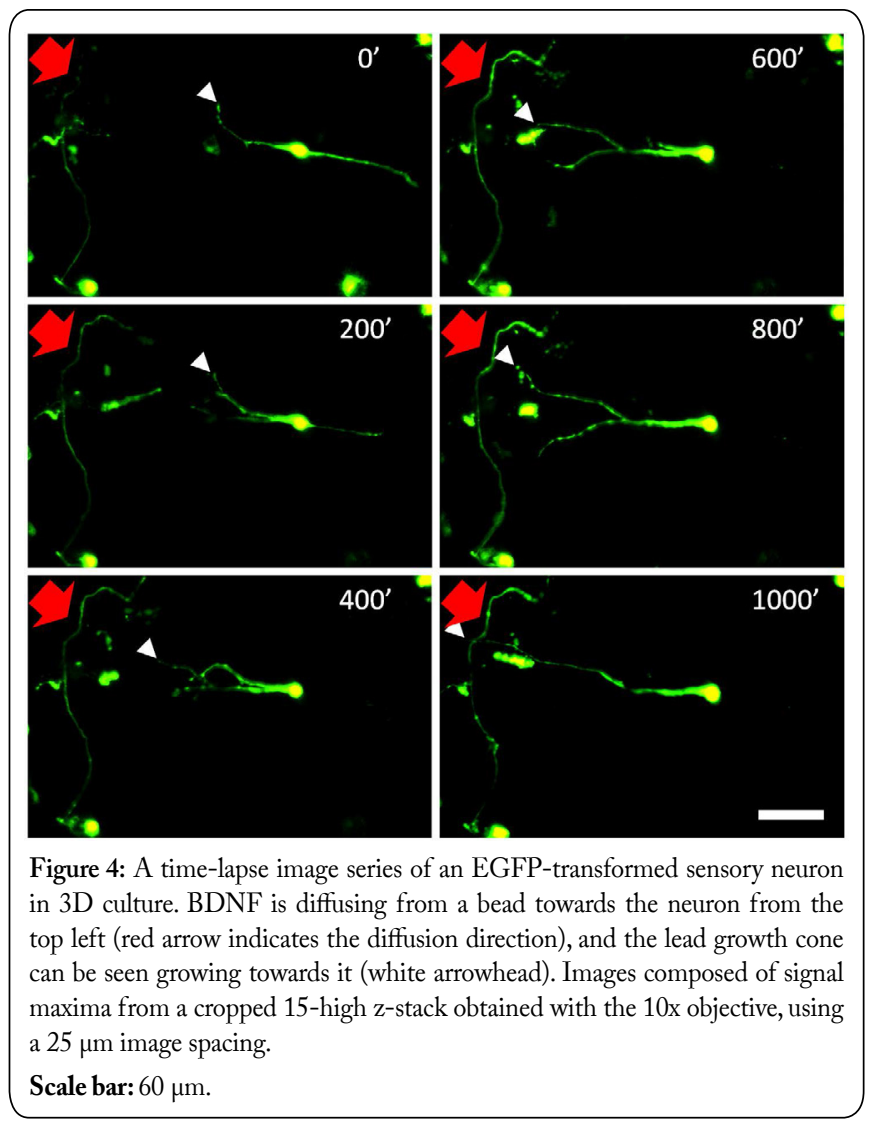


molecule-soaked beads, and the subsequent initiation of time-lapse image recording with one or more neurons in focus (Figure 4). This method provides a way of analysing the interaction between single neurons and a test molecule point-source, including growth rate, morphology and growth cone behaviour. Specifically, using fluorescent tubulin and actin constructs might yield clues to the exact mechanisms of cytoskeletal rearrangement in play in a growth cone responding to a chemoattractive/repulsive gradient. This has only now become possible due to the very high spatial and temporal resolution of modern spinning disc confocal microscopy.

In conclusion, these methods provide a modern means to visualize, record and analyse how neurons respond to various long-range signalling factors in vitro. They span a range of complexities, costs and uses, with software-aided analysis available for bulk data collection and the statistical analysis of results. We hope that their implementation will aid the discovery of new neurotrophic factors, or identify neurotrophic effects in proteins previously thought unrelated to nervous system development, such as VEGF [22]. Exploitation of their axon guidance and growth stimulating properties, along with studying their expression regulation mechanisms, could pave the way for novel neuroregenerative therapies.

\section{Acknowledgements}

The authors would like to thank A. Lodwig, C. Grzelak and S. Wenderdel for their technical assistance. We would also like to give special thanks to the Claussen-Simon Stiftung.

\section{References}

1. Paralysis facts \& figures - Spinal cord injury - Paralysis Research Center.

2. Siemionow M, Brzezicki G. 2009. Chapter 8: Current techniques and concepts in peripheral nerve repair. Int Rev Neurobiol 87: 141-172. doi: 10.1016/S0074-7742(09)87008-6

3. Waller A. 1850. Experiments on the section of the glossopharyngeal and hypoglossal nerves of the frog, and observations of the alterations produced thereby in the structure of their primitive fibres. Philosophical Transactions of the Royal Society of London 140: 423-429.

4. O'Donnell M, Chance RK, Bashaw GJ. 2009. Axon growth and guidance: receptor regulation and signal transduction. Annu Rev Neurosci 32: 383-412. doi: 10.1146/annurev.neuro.051508.135614

5. Hanz S, Perlson E, Willis D, Zheng JQ, Massarwa R, et al. 2003. Axoplasmic importins enable retrograde injury signaling in lesioned nerve. Neuron 40(6): 1095-1104. doi: 10.1016/S0896-6273(03)00770-0

6. Wujek JR, Lasek RJ. 1983. Correlation of axonal regeneration and slow component B in two branches of a single axon.J Neurosci 3(2): 243-251.

7. $\mathrm{Ma} \mathrm{CH}$, Omura $\mathrm{T}$, Cobos EJ, Latrémolière $\mathrm{A}$, Ghasemlou $\mathrm{N}$, et al.
2011. Accelerating axonal growth promotes motor recovery after peripheral nerve injury in mice. J Clin Invest 121(11): 4332-4347. doi: 10.1172/JCI58675

8. Brushart TM, Mesulam MM. 1980. Alteration in connections between muscle and anterior horn motoneurons after peripheral nerve repair. Science 208(4444): 603-605. doi: 10.1126/science.7367884

9. Baum CM, Weissman IL, Tsukamoto AS, Buckle AM, Peault B. 1992. Isolation of a candidate human hematopoietic stem-cell population. Proc Natl Acad Sci U S A 89(7): 2804-2808.

10. Birbrair A,ZhangT,Wang ZM, Messi ML, Enikolopov GN, et al.2013. Role of pericytes in skeletal muscle regeneration and fat accumulation. Stem Cells Dev 22(16): 2298-2314. doi: 10.1089/scd.2012.0647

11. Tumbar T, Guasch G, Greco V, Blanpain C, Lowry WE, et al. 2004. Defining the epithelial stem cell niche in skin. Science 303(5656): 359363. doi: $10.1126 /$ science. 1092436

12. Deister C, Schmidt CE. 2006. Optimizing neurotrophic factor combinations for neurite outgrowth. J Neural Eng 3(2): 172-179. doi: $10.1088 / 1741-2560 / 3 / 2 / 011$

13. McMahon SB, Armanini MP, Ling LH, Phillips HS. 1994. Expression and coexpression of Trk receptors in subpopulations of adult primary sensory neurons projecting to identified peripheral targets. Neuron 12(5): 1161-1171. doi: 10.1016/0896-6273(94)90323-9

14. Campenot RB. 1977. Local control of neurite development by nerve growth factor. Proc Natl Acad Sci U S A 74(10): 4516-4519.

15. Lumsden AG, Davies AM. 1986. Chemotropic effect of specific target epithelium in the developing mammalian nervous system. Nature 323(6088): 538-539. doi: 10.1038/323538a0

16. Joanne Wang C, Li X, Lin B, Shim S, Ming GL, et al. 2008. A microfluidics-based turning assay reveals complex growth cone responses to integrated gradients of substrate-bound ECM molecules and diffusible guidance cues. Lab Chip 8(2): 227-237. doi: 10.1039/ b713945d

17. Dupin I, Dahan M, Studer V. 2013. Investigating axonal guidance with microdevice-based approaches. J Neurosci 33(45): 17647-17655. doi: 10.1523/JNEUROSCI.3277-13.2013

18. Baker BM, Chen CS. 2012. Deconstructing the third dimension: how 3D culture microenvironments alter cellular cues. J Cell Sci 125(pt3): 3015-3024. doi: 10.1242/jcs.079509

19. Ribeiro A, Vargo S, Powell EM, Leach JB. 2012. Substrate threedimensionality induces elemental morphological transformation of sensory neurons on a physiologic timescale. Tissue Eng Part A 18(1-2): 93-102. doi: 10.1089/ten.tea.2011.0221

20. Masyuk M, Abduelmula A, Morosan-Puopolo G, Ödemis V, Rehimi $\mathrm{R}$, et al.2014. Retrograde migration of pectoral girdle muscle precursors depends on CXCR4/SDF-1 signaling. Histochem Cell Biol 142(5): 473488. doi: 10.1007/s00418-014-1237-7

21. Chamber gradient stability.

22. Sondell M, Lundborg G, Kanje M. 1999. Vascular endothelial growth factor has neurotrophic activity and stimulates axonal outgrowth, enhancing cell survival and Schwann cell proliferation in the peripheral nervous system. J Neurosci 19(14): 5731-5740. 